\title{
Ein Fall von hämorrhagischer Adenie mit symmetrischen Lymphomen der Bindehaut.
}

\author{
Von \\ Prof. Dr. W. Goldzieher \\ in Budapest.
}

Ein Fall von symmetrischem Lymphom der Bindehäute, der sowohl von den mir aus eigener Erfahrung bekannten, als auch von den in der Literatur beschriebenen in vielen und wesentlichen Stücken abwich, möge hier in Kürze beschrieben werden.

Lymphomatöse Wucherung der Bindehaut im Verein mit weitverbreiteter Hyperplasie von Lymphdrüsen wird in der Regel mit einer der leukämischen oder pseudoleukämischen Allgemeinerkrankungen in Verbindung gebracht. In dem zu beschreibenden Falle kann trotz mancher äusserer Ähnlichkeiten keine der beiden Annahmen aufrecht erhalten werden; wir finden im Gegenteil eine Reihe von Merkmalen, die ihn zu einem neuen, bisher noch unbekannten Krankheitsbilde stempeln.

\section{Krankengeschichte.}

Es handelt sich um einen 45jährigen Fuhrmann, V. S. aus Also-Ròna in Ungarn, der bis vor vier Jahren vollkommen gesund war. Er stammt aus gesunder Familie, ist verheiratet, hat fünf lebende, vollkommen gesunde Kinder. Er behauptet immer mässig gewesen zu sein und niemals an einer Geschlechtskrankheit gelitten zu haben.

Vor vier Jahren will er durch einige Tage Augenschmerzen gehabt haben. Seit ungefähr zwei Jahren soll die Verbärtung um die Augen bestehen; vor 8 Monaten soll das rechte Auge beträchtlich angeschwollen sein.

Schon auf den ersten Anblick bietet der Kranke ein eigentümliches Bild. Die Gesichtshant ist mässig cyanotisch, von zahlreichen ausgedehnten und geschlängelten Hautvenen durchsetzt. Der Kranke macht den Eindruck eines Schläfrigen, da die Augenlider halb geschlossen sind und auch auf Aufforderung nicht weit geöffnet werden können. Die Haut der Augenlider, namentlich rechterseits, ist straff gespannt, die Falten 
wie ausgeglichen machen den Eindruck, wie wenn sie von innen her nach vorne gepresst wären. In der Tat sehen wir auch Anschwellungen rechterseits längs des oberen und unteren Orbitalrandes, linkerseits nur längs des unteren Orbitalrandes.

Die genauere Untersuchung der Augen ergibt folgendes:

Linkes Auge: Das obere Lid ist leicht umzustülpen. Die Conjunctiva tarsi ist in hohem Grade gerötet, wie himbeergeleefarbig, das Gefässnetz prall gefüllt, mit deutlich sichtbaren ausgedehnten und geschlängelten Gefässen. Die Bindehaut ist sonst glatt, zeigt weder eine papilläre noch follikuläre Schwellung. Die obere thbergangsfalte ist normal. Das untere Lid ist jedoch nur mit einiger Gewalt nach unten zu rollen, nach der Abziehung springt die untere Übergangsfalte in ihrer ganzen Länge in Gestalt einer mächtigen, walzenförmigen, auf der Oberfläche glatten, tief dunkelroten Geschwulst hervor. Die Conjunctiva tarsi ist abgesehen von der hochgradigen Injektion normal. Conjunctiva bulbi oberflächlich injiziert; Hornhaut klar; vordere Kammer von normaler Tiefe; die Vorderfläche der Iris von normalem Gefüge; Pupille eng, winklig, zahlreiche hintere Synechien, durch Atropin nicht zu erweitern, im Pupillargebiete eine zarte, hauchförmige Pseudomembran. Linse scheint von normaler Durchsichtigkeit zu sein; aus dem Augenhintergrund ist nur rotes Licht zu bekommen. V. ungefähr 0,1 (Analphabet!).

Rechtes Auge: Das obere Lid ist stark gespannt, ein wenig ödematös. Längs des oberen Orbitalrandes ist eine unter dem Lide zu fühlende harte, walzenförmige Geschwulst, die in transversaler Richtung ein wenig hin- und hergeschoben werden kann. Das obere Lid kann nur mit Mühe umgestülpt werden, und auch dann nicht vollkommen, worauf man den unteren Rand der erwähnten Geschwulst zu Gesichte bekommt. Das untere Lid kann jedoch vollständig abgerollt werden, worauf, wie linkerseits, der in eine dunkelrote Geschwulst verwandelte untere Fornix vorspringt. Die übrige Bindehaut wie links. Am Bulbus bis auf einige punktförmige hintere Synechien nichts abnormes zu sehen. Augenhintergrund normal. Sehschärfe (Zeichentafel) gut.

Die prä- und retroaurikulären Lymphdrüsen sind beiderseits, jedoch in grösserem Masse rechts, zu grossen Paketen angeschwollen. Die Parotis scheint beiderseits normal zu sein. Die Achseldrüsen sind nicht vergrössert; in der linken Leistenbenge wölbt sich eine ungefähr gänseeigrosse, harte, gut verschiebliche Geschwulst hervor; in der rechten Leistengegend sind zahlreiche kleinere, mit einander nicht verwachsene Drüsengeschwülste $\mathrm{zu}$ fühlen.

Am Brustkorbe ist weder durch Perkussion, noch durch Auskultation eine Abweichung von der Norm wahrzunehmen. Höchstens dass in der rechten Paravertebrallinie, neben den IV. bis VI. Wirbeln, der Perkussionsschall in geringem Grade gedämpft ist.

Bauchorgane normal. Keine Milz- und Lebervergrösserung.

Die Röntgenuntersuchung, die hauptsächlich wegen der Dämpfung in der rechten Paravertebrallinie vorgenommen wurde, ergibt nichts erwähnenswertes. 
Ein Fall von hämorrhagischer Adenie mit symmetrischen Lymphomen usw.

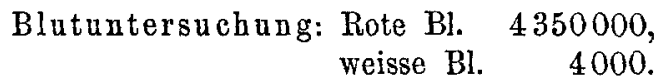

Im gefärbten Präparate sind sehr wenig Lymphocyten, in etwas grösserer Menge polynucleare Leukocyten zu sehen.

Therapie und Verlauf: Innerlich wird Solutio arsenic. Fowl. gegeben. Am 11.XI. 1906 wird die Geschwulst der linken unteren Übergangsfalte entfernt. Nach maximaler Abrollung des unteren Lides wurde die vorspringende Geschwulst mit der Hakenpincette gefasst und mit der krummen Schere rein abgetragen. Nach der Abtragung wird durch sorgfältiges Tasten festgestellt, dass im retroconjunctivalen Gewebe weder Stränge, noch Geschwulstlinoten vorhanden sind. Naht unnötig. Einfacher Verband.

14. XI. 1906. Mit Einwilligung des Patienten wird hauptsächlich zur histologisehen Untersuchung die Lymphdrüsengeschwulst der linken Leistenbeuge unter Schleichscher Anästhesie glatt und leicht ausgelöst. Heilung vollkommen. Die Fäden werden am 21. XI. entfernt.

24. XI. 1906. Da die freie Bewegung der rechtsseitigen Lider wegen der Fornixgeschwülste sebr behindert ist, so wird die Exstirpation vorgeschlagen. Der Kranke gestattet jedoch keinen grösseren Eilgriff mehr (wegen der Spannung des Oberlides hätte zur Entfernung der grossen oberen Fornixgeschwulst eine ausgiebige Erweiterung der Lidspalte gemacht werden müssen), und gibt schliesslich die Erlaubnis zur Entfernung der unteren Fornixgeschwulst, die ebenso wie an der linken Seite leicht gemacht werden konnte.

29. XI. Die Wunde der unteren Übergangsfalte linkerseits ist vollkommen, mit geringer Verkürzung des Bindehauttractus geheilt. Auch rechterseits vollzieht sich die Heilung obne Zwischenfall.

Die Geschwulst des rechten oberen Fornix, sowie der Lymphdrüsen ist trotz des Arsengebrauchs unverändert geblieben.

Blutuntersuchung vor der Entlassung am 29. XI.: Hämoglobingehalt 67, rote Bl. 3400000 , weisse Bl. 6400 . Die beträchtliche Abnahme der Erythrocyten wird damit erklärt, dass der Kranke, der als Fubrmann in einer Gebirgsgegend lebt und an andere als die Spitalskost gewöhnt war, sich im Spitale sehr elend fühlte und seine Esslust verlor.

Über sein weiteres Schicksal unterrichtet mich ein anfangs Juli d. J. eingelaufener Brief, wonach er sich sehr wohl befindet und sein Geschäft ohne Störung versieht. Leider wird in dem von fremder Hand geschriebenen Berichte nichts über den Zustand der Lymphdrüsen gesagt.

Untersuchung der exstirpierten Geschwülste.

Die ausgeschälte Inguinaldrüse, ungefähr von der Grösse eines Gänseeies, fiel schon dem freien Auge dadurch auf, dass ihre Obertläche tief dunkelrot gefärbt war, ihre Konsistenz war prall elastisch, ihre Schnittfläche succulent, gesprenkelt, indem Stellen von hellerem Rot mit augenscheinlich hämorrhagischen abwechselten.

Ein ähnliches Bild boten die entfernten Fornixgeschwülste, indem auch bei ihnen der hyperämisch-hämorrhagische Charakter auf der Durchschnittsfläche ersichtlich war. 
Was die Inguinaldrüse anbelangt, so liessen sich mikroskopisch vor allem äusserst zahlreiche Blutungen im Drüsenparenchym nachweisen, die durch ihr fleckweises Auftreten das gesprenkelte Aussehen der Sehnittfläche hervorriefen.

Das Drüsenparenchym selbst besteht fast ausschliesslich aus kleinen, runden, mononucleären Leukocyten, zwischen denen Lymphocyten mit noch kleineren dunkler gefärbten Kernen und spärlichem Protoplasma nur vereinzelt vorkamen. Auffallend sind im histologischen Bilde stellenweise auftretende Herde wechselnder Grösse, die schon bei schwacher Vergrösserung durch ihre homogene Beschaffenheit in die Augen fallen. Diese Herde sind nun, wie stärkere Systeme zeigen, aus einer sich schwach mit Eosin, nicht mit Fuchsin S. färbenden Masse gebildet, in der sich zahlreiche epitheloide Zellkerne von teils ovaler, teils rundlicher oder gelappter Form vorfinden. Sie färben sich äusserst schwach und lassen in der sie umgebenden homogenen Masse nur undeutlich ihren Zellleib erkennen.

Diese, Zellherde sind meist von rundlicher, knötchenartiger Form, liegen streng umschrieben im Drüsenparenchym zerstreut and sind von dicht gedrängten Parenchymzellen wie von einer Kapsel begrenzt.

Ein weiterer wichtiger Befund sind vereinzelte Riesenzellen typischer Langhansscher Art mit randständigen Kernen und krümeligen, wie verkästem zentralen Protoplasma. Dieser Befund konnte jedoch nur an zweien unter den zahlreichen andern Schnitten erhoben werden, trotz sorgfältigen Nachsuchens. Beide Male lagen die Riesenzellen ganz frei im Drüsenparenchym und standen in keinerlei nachweisbarer Beziebung zu den in der Nähe befindlichen Anhäufungen von Epitheloidzellen.

Was das histologische Bild der Conjunctivalgeschwïlste betrifft, so ist es für beide Augen identisch und zeigt auch im wesentlichen - wenn man die Verschiedenheit des Grundgewebes berücksichtigt - viele Übereinstimmung mit dem Befund des Inguinallymphoms. Unter den wenig veränderten, nicht in die Tiefe gewucherten Epithelien des Fornix finden wir eine, die Hauptmasse der Geschwulst ausmachende dichte Anhäufung von meistens mononucleären Leukocyten, mit zahlreich eingestreuten Lymphocyten, in der strotzend gefüllte Gefässe sowie reichliche Blutergüsse anzutreffen sind. Dagegen konnten keine Epitheloidzellenknötchen gefunden werden, wenn anch einzelne Epitheloidzellen häufig vorkommen. Riesenzellen waren in keinem einzigen Präparate zu finden.

Die Untersuchung auf Tuberkelbacillen blieb negativ.

Das Studium der mikroskopischen Präparate ergab demnach mit Bestimmtheit, dass eine Adenie von hämorrhagischem Charakter vorliegt. Über die Natur des Krankheitserregers jedoch kann keine positive Angabe gemacht werden.

Man könnte nach dem Befunde versucht sein, eine Form von tuberkulöser Entzündung anzunehmen; dafür scheinen die Anhäufungen epitheloider Zellen, oft in Knötchenform, ferner die, wenn auch äusserst spärlichen typischen Riesenzellen zu sprechen. Dagegen aber sprechen, bei der langen Dauer des Prozesses, der Mangel der regressiven Veränderungen, ferner der Umstand, dass nirgends Verkäsung zu finden und die 
Drüsengewebshyperplasie sehr stark ansgeprägt ist, sowie der riesige Blutreichtum und die ausgedehnten Blutungen, einigermassen ferner noch der eminent gutartige Verlauf.

Wenn wir nun vom klinischen Standpunkte die Hauptmerkmale des vorliegenden Krankheitsfalles zusammenfassen, so muss auf folgende besonderes Gewicht gelegt werden:

1. Die Spuren vorangegangener Iritis, die rechterseits mit wenig Synechien geendet, linkerseits aber Pupillenverlötung erzeugt hat.

2. Weitverbreitete lymphomatöse Geschwülste bei völligem Freibleiben der Leber und Milz.

3. Lymphomatöse Verbildung beider Übergangsfalten der Conjunctiva am rechten Auge und der unteren Ü̈bergangsfalte links, bei vollkommener Intaktheit des Orbitalgewebes und speziell der Tränendrüse.

Was den ersten Punkt anbelangt, so wissen wir allerdings nicht, ob die Iritis zur Adenopathie in irgend einer ursächlichen Verbindung steht, oder ob es sich um ein zufälliges Zusammentreffen handelt. Diese Iritis, über deren Verlauf wir vollkommen im unklaren sind, deren Beginn wir nur vermutungsweise auf vier Jahre vor der Beobachtungszeit setzen können, muss jedenfalls sehr schleichend und ohne viel Schmerzen zu machen verlaufen sein. Da aber weder für Syphilis, Gonorrhoe, Rheumatismus Anhaltspunkte vorhanden sind, so liegt der Gedanke nahe, dass derselbe Krankheitserreger, der die Iritis verursachte, auch für das Drüsenleiden verantwortlich zu machen ist.

Betreffs der Drüsengeschwïlste ist zu bemerken, dass ein wesentliches Merkmal vorhanden ist, welches sie streng von den leukämischen und pseudoleukämischen unterscheidet. Es ist dies ihr hyperämisch - hämorrhag is cher Charakter. Die Drüsengeschwülste lenkämischer und pseudoleukämischer Herkunft haben, wie die pathologische Anatomie lehrt, eine weisse oder grauweisse, nur sehr selten eine graurote Schnittfiäche ${ }^{1}$ ). Harte Lymphome sind direkt von gelblicher Farbe, von derber, trockener Schnittfläche. Von dieser Schilderung sticht lebhaft die hier beschriebene, auf der Oberfäche dunkelrote, auf der Schnittfläche succulente und hämorrhagische Inguinaldrüsengeschwulst ab. Tch stehe nicht an, zur Kennzeichnung der vorliegenden Geschwulst den Vergleich mit dem Pestbubo heranzuziehen, der sich bekanntlich durch seinen exquisit hämorrhagischen

1) Ziegler, Lehrb. d. allgem. Pathol. u. pathol. Anat. 1906, S. 146. 
Charakter ausgezeichnet. Auf diesen etwas überraschenden, aber treffenden Vergleich wurde ich durch Professor E. Krompecher aufmerksam gemacht, dem ich die frisch exstirpierte Drüse zeigte: Er ermächtigte mich auch, hier anzuführen, dass diese Drüse durchaus jenen gleiche, die von Professor O. Pertik auf einer Studienreise zur Erforschung der Bubonenpest für das Budapester pathol.anat. Museum gesammelt und in vorzüglichen farbigen Abbildungen zu Lehrzwecken reproduziert wurden. Ebenso wie der Inguinaltumor, so besassen auch denselben hämorrhagischen Charakter, wie oben geschildert, die exstirpierten Fornixgeschwiilste.

Was diese betrifft, möchte ich, um die vorliegenden Veränderungen so scharf als möglich hervorzuheben, betonen, dass sie durchaus von andern, in der Literatur beschriebenen, mit Lymphomen der Halsdrüsen sich komplizierenden verschieden sind. Es bedarf vor allem kaum einer besonderen Erwähnung, dass es sich hier nicht um eine Conjunctivitis mit Lymphomen ${ }^{1}$ ) (von mir als „Lymphom-Conjunctivitis", zumeist aber als Parinaudsche Krankheit bezeichnet) handelt. Diese Krankheit ist einseitig, betrifft die gesamte Lidbindehaut und zeigt sich in der Form sehr üppig wachsender Granula und hahnenkammähnlichen Excrescenzen, gleichsam ein ins riesenmässige entwickelte Trachom vortäuschend. Auch ist mir kein Fall bekannt, wo bei dieser Krankheit ausser den Halsdrüsen an der Seite des affizierten Auges noch andere Lymphdrüsen ergriffen worden wären. Im vorliegenden Falle war auf der Bindehant keine Spur einer entzündlichen Veränderung vorhanden, ausschliesslich die Übergangsfalten waren geschwulstartig degeneriert.

Die lymphomatöse Erkrankung der Bindehaut, wie sie, wenn auch nicht sehr häufig, bei Pseudoleukämie beobachtet wird, von der ein klassischer Fall von Axenfeld ${ }^{2}$ ) beschrieben wurde, stellt sich ebenfalls unter einem andern Bilde dar. In dem Axenfeldschen Falle war die Oberfläche der Conjunctivalgeschwülste graugelblich, glasig, speckig. Die linke obere Übergangsfalte glich einem kolossalen sulzigen Trachomwulst; auch waren in der Orbita ähnliche Knoten zu fühlen. In meinem Falle ist trotz mehrjährigem Bestehen der Fornixtumoren die Conjunctiva tarsi normal und das Orbitalgewebe von Tumormassen vollkommen frei geblieben. Auch der Fall V. $\left.B e r l s^{3}\right)$, der zweifellos zur pseudoleukämischen Gruppe gehört,

1) Zentralbl. f. prakt. Augenheilk. 1905, S. 1.

$\left.{ }^{2}\right)$ Axenfeld, Arch. f. Ophth. Bd. XXXVI, 4, S. 102-124.

$\left.{ }^{3}\right)$ Beitr. zur Augenheilk. 1899. Heft XXXVII, S. 32-46. 
unterscheidet sich wesentlich von dem vorliegenden. In jenem waren die Geschwülste subconjunctival gelegen und die Übergangsfalten, wenn auch stellenweise mit ihnen verwachsen, nur sulzig degeneriert. In einem neueren Falle von $\mathrm{O} . \mathrm{Napp}^{1}$ ), wo es sich um eine Art Mikuliczscher Erkrankung handelt - eine Krankheitsgruppe die übrigens erst durch die iibersichtliche Arbeit J. Mellers ${ }^{2}$ ) ihre richtige Beleuchtung erfahren hat -, befanden sich miliare Knötchen und Excrescenzen im Bindehauttractus, die sich bei der Untersuchung als tuberkulös erwiesen. Ähnlichkeit mit meinem Falle scheint eine Beobachtung Guaitas aus dem Jahre $1890 \mathrm{zu}$ haben, den ich leider nur unvollständig aus einem Referate des Zentralbl. f. prakt. Augenheilk. (Bd. XIV, S. 557) kenne. Hier war neben weit verbreiteten Lymphdrüsenschwellungen noch Milzschwellung und eine Verdickung der Ubergangsfalten vorhanden. Die Conjunctiva blutete beim Umstuilpen leicht, sie war aber glatt und blass. Ausgeschnittene Stückchen der Geschwulst zeigen reichliche Lymphzelleninfiltration. Ein hämorrhagischer Charakter der Bindehautgeschwulst scheint jedoch nicht vorhanden gewesen zu sein, sonst müsste, so sollte man meinen, die histologische Beschreibung auch im Referate doch eine Andeutung hiervon enthalten. Eben dieser hämorrhagische Charakter, der gleichmässig im Lymphdrüsentumor als auch in den Conjunctivallymphomen herrortritt, ist es, was den beschriebenen Fall einer besonderen Beachtung für wert erscheinen lässt.

1) Napp, Zeitschr. f. Augenheilk. 1907, Juni-Heft, S. 513.

2) J. Meller, Über die Beziehungen der Mikuliczschen Erkrankung zu den lymphom. u. chron. entzündl. Prozessen. Klin. Monatsbl. f. Augenheilk. 1906, Sept.'Siehe ferner die Arbeit desselben Autors über: Die lymphom. Geschwulstbildungen in der Orbita und im Auge. Arch. f. Ophth. Bd. LXII, 1, wo die Literatur zusammengestellt ist. $\mathrm{Da}$ ich mich hier nur auf das notwendige beschränke, so sei ausdrücklich auf diese wichtige Arbeit aufmerksam gemacht. 